\title{
PEPPER: PolARIZATION-ENCODING DIFFERENTIAL Photometer and Polarimeter
}

\author{
MASTER's REPORT \\ BY \\ Catarina Coutinho Ubach
}

JUNE 28, 2009

A Document Submitted to the Faculty of the

College of Optical Sciences

In Partial Fulfillment of the Requirements

For THE DEGREe OF

Master of SCIENCE

In the Graduate College

THE UNIVERSITY OF ARIZONA 


\section{ACKNOWLEDGMENTS}

I would to take the opportunity to thank the people that helped me. I thank Dr. Dan Potter for allowing me to write about PEPPER, and taking the time to correct this report. I also want to thank Prof. Chipman for being my masters report advisor, and one of the committee members. I also want to recognize the other two committee members, Prof. Tyo, and Prof. Dereniak.

I want to thank my family and friends for their support. I also want to thank Tim Schnauber for helping me with LaTex.

Thank you all 


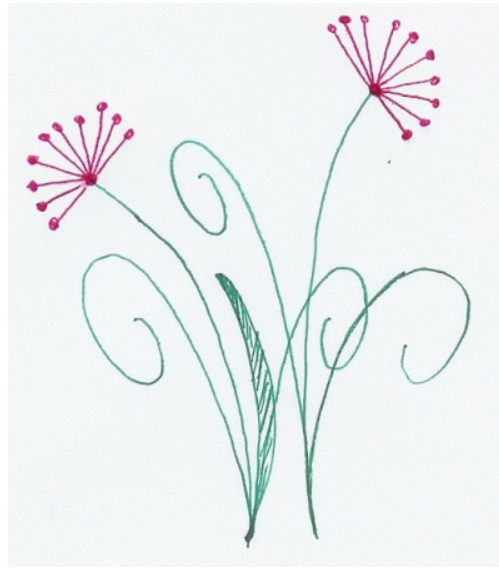

For those who love me. 


\section{Table of Contents}

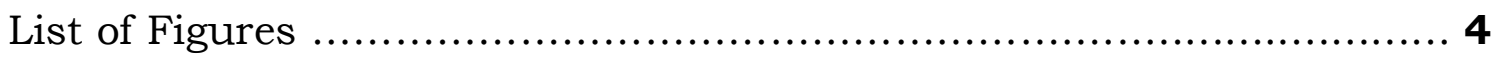

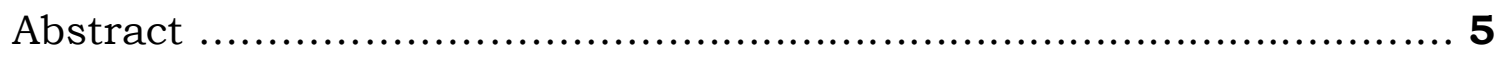

Chapter 1: PEPPER........................................................ 6

1.1 Polarimeter...................................................... 6

1.2 Photometer................................................... 11

Chapter 2: Instrument Applications.................................. 16

2.1 Polarimetry: Data Reduction and Analysis..................... 18

2.2 Photometry: Data Reduction and Analysis...................... 25

Conclusion.......................................................... 30

References............................................................ 31 


\section{List OF Figures}

FIGURE 1.1. PEPPER . . . . . . . . . . . . . . . . . . . . 7

Figure 1.2. PEPPER at the telescope . . . . . . . . . . . . . . 8

Figure 1.3. Polarimeter . . . . . . . . . . . . . . . . . . . . . . 9

Figure 1.4. Raw Data . . . . . . . . . . . . . . . . . . . . . . 10

Figure 1.5. Wollaston Prism . . . . . . . . . . . . . . . . . . 11

Figure 1.6. Polarimeter Schematic . . . . . . . . . . . . . . . . . . 12

Figure 1.7. Photometer . . . . . . . . . . . . . . . . . . . . . 13

Figure 1.8. Polarization Encoded Optics . . . . . . . . . . . . . . . . . . 15

Figure 2.1. ROXR1-35 . . . . . . . . . . . . . . . . . . . . . . . . 20

Figure 2.2. ROXR1-35 image with polarization . . . . . . . . . . . . . . . . 22

Figure 2.3. ROXR1-35 final image . . . . . . . . . . . . . . . . . . 23

Figure 2.4. Polarization Analysis . . . . . . . . . . . . . . . . . . . 24

Figure 2.5. ROX34: No Detection of disk . . . . . . . . . . . . . . . . 25

Figure 2.6. HD209458 Transit . . . . . . . . . . . . . . . . . . 26

Figure 2.7. Model of HD209458b . . . . . . . . . . . . . . . . . . . . . . . 27

Figure 2.8. Sample Image of HD209458b . . . . . . . . . . . . . . . . . . . 28

Figure 2.9. Differential Signal for HD209458b . . . . . . . . . . . . . . . . . 29 


\begin{abstract}
PEPPER is a high-speed differential Polarization-Encoded Photometer and Polarimeter developed in the Center for Astronomical Adaptive Optics at the University of Arizona, Tucson, by Dr. Dan Potter and Matthew Graham. PEPPER is capable of acting as a high-speed polarimeter by using electro-optical switching to chop between standard star and target star, and between in and out-feature bandpass filter at frequencies fast enough to suppress atmospheric variations (1). PEPPER is capable of either high-speed polarimetry or differential photometry using a combination of simultaneous imaging and electro-optical switching. In the differential photometry mode, PEPPER utilizes the electro-optical switching to calibrate instrumental and atmospheric photometric variation. This technique coupled with a zero-read noise photon counting detector achieves photon noise limited results demonstrated to an accuracy of less than 1 part in $10^{5}$. I will present the design concept behind the photometer and the polarimeter mode of PEPPER, as well as, results from observations in the differential photometer mode at the Steward 90 inch telescope, at the Kitt Peak National Observatory, Tucson, Arizona. Results from the analysis of near IR polarimetry observations of young stars with circumstellar disks taken at the Gemini North Telescope with the Hokupa'a adaptive optics system are also presented.
\end{abstract}




\section{Chapter 1 PEPPER}

PEPPER (Polarization-Encoded differential Photometer and PolarimetER) (3) was designed to detect light from phase change closed-in eclipsing extra-solar planets, and faint circumstellar debris disks (2). As it will be showed in this report, PEPPER can achieve near photon noise limit results in the laboratory and on sky observations to levels of 1 part in $10^{5}$. This capability allows for atmospheric variation and detector noise suppression, which gives PEPPER the ability of recording high resolution data without the use of adaptive optics and a space telescope, which is an advantage when doing groundbase astronomy. The instrument was first developed in 2004 by Dr. Dan Potter and Matthew Graham at the Center for Astronomical Adaptive Optics, Steward Observatory, University of Arizona, Tucson. After initial testing done at the Vatican Telescope on Mt. Graham, some modifications have been made to lower the noise to acceptable levels. This chapter of the report describes the latest model of the PEPPER.

\subsection{Polarimeter}

The Polarimeter was used to detect circumstellar disks around a star(s). PEPPER consists of a 100 pounds casing, enclosing all the optical instruments (figure 1.1). This casing was attached to the bottom of the Bok telescope and the computer attached to the outside of the casing (figure 1.2 and figure 1.3). This allows for the remote setup of the camera, and a display of what is being observed. The artificial light source is used during the differential photometric observations (that is the "standard star" c1,2 in figure 1.4).

The camera read out was at $\sim 120 \mathrm{~Hz}$ and the Ferro-electric Liquid Crystals, FLC, modulator was going at $\sim 30-50 \mathrm{~Hz}$. The artificial light source calibrated the actual state of the FLC. The FLC were used as an achromatic switchable wave plate, which did not introduce any polarization but acted as a variable retarder (shifts the phase by $1 / 2$ a wave when it is turned on) that worked over a broad wavelength 


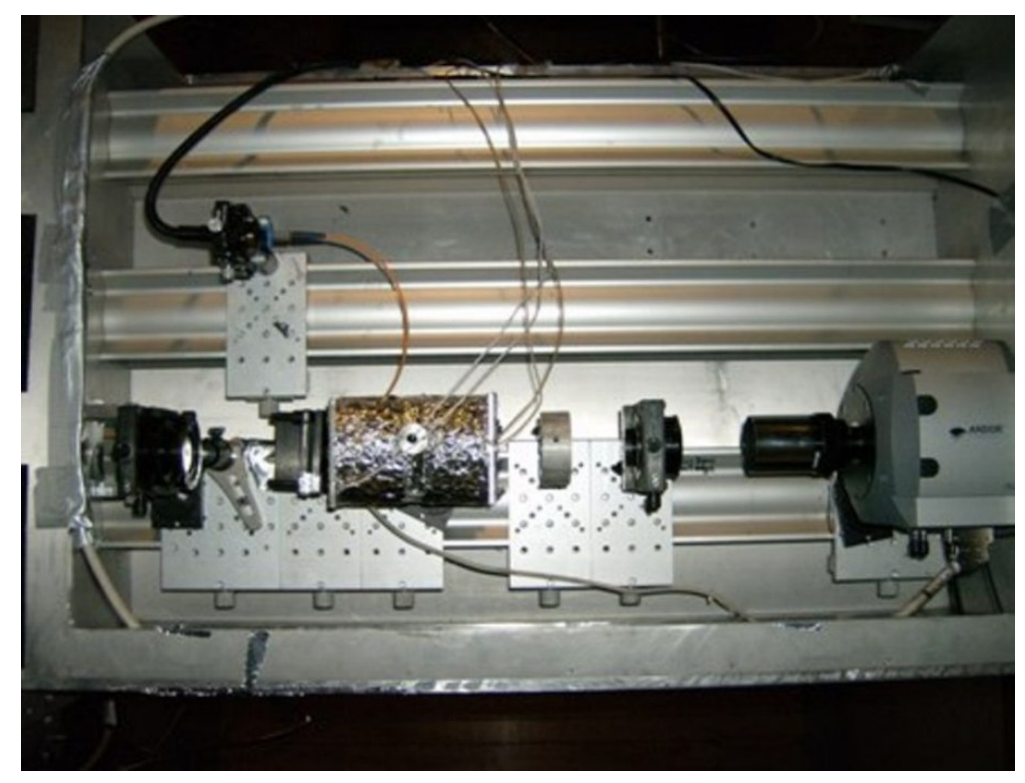

Figure 1.1. Polarization Encoding differential Photometer and PolarimetER, PEPPER, mounted at Bok Telescope.

bandwidth. The FLC has to be $1 / 2$ of the frequency of the camera so each state can be imaged. The light of the object then passes through the Wollaston Prism. The Wollaston Prism then separates unpolarized light or randomly polarized light into two orthogonal outgoing linearly polarized beams. The outgoing light beams diverge at $1.5^{0}$ from the prism with $\mathrm{s}$ and p polarizations figure $1.5(2)$.

The light is then focused by the focusing lens into a sensitive Electron Multiplying Charge-Coupled Device (EMCCD) that enables fast modulation (7). The observer will see two images, one of the s polarization of the object, and the second, of the $\mathrm{p}$ polarization of the object (figure 1.5 and 1.6).

The raw images are acquired having the EMCCD set at $120 \mathrm{~Hz}$ per second, in the course of 8 minutes; about 60,000 images can be taken to create an image cube of the object being observed. The atmospheric effects are taken out with the simultaneous imaging of the s and p by the Wollaston prism. The fast modulation enables speckle reconstruction techniques to be used to achieve better spatial resolution. The modulation also reduces the noise associated with instrument gain variations. These raw images are then reduced and analyzed as described on chapter 2 to determine whether or not the star(s) possesses a circumstellar disk. When circumstellar disks 


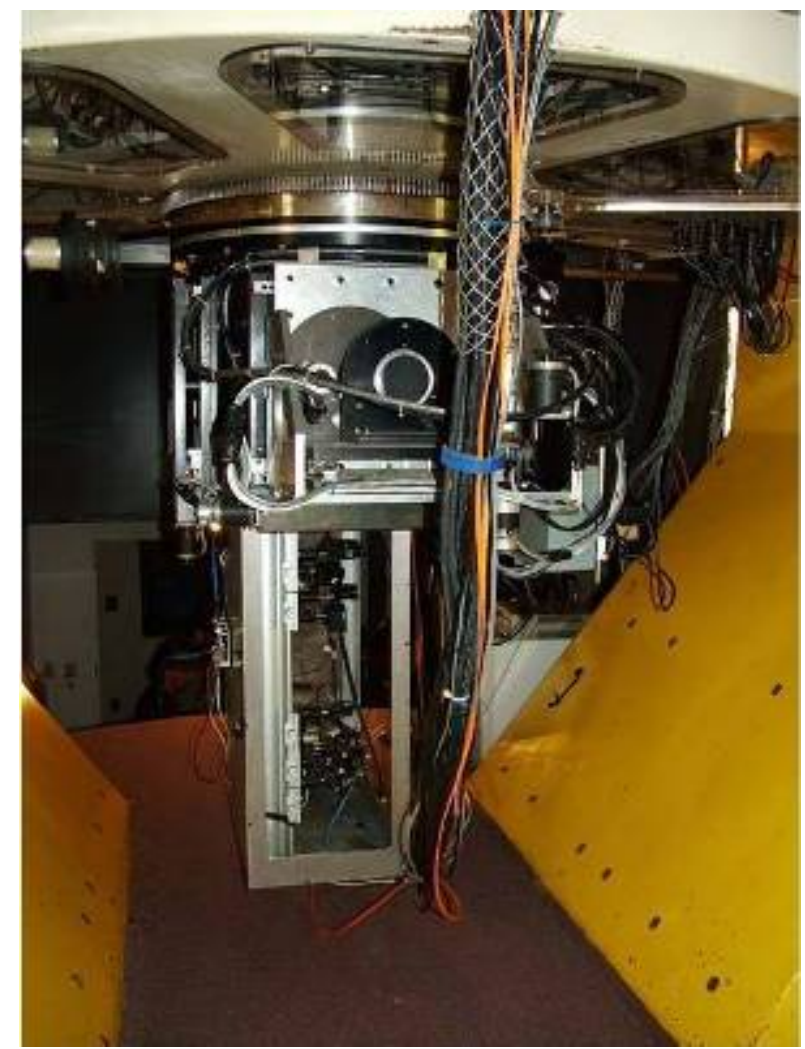

Figure 1.2. PEPPER attached to a telescope. (Picture provided by Dr. Dan Potter.) 


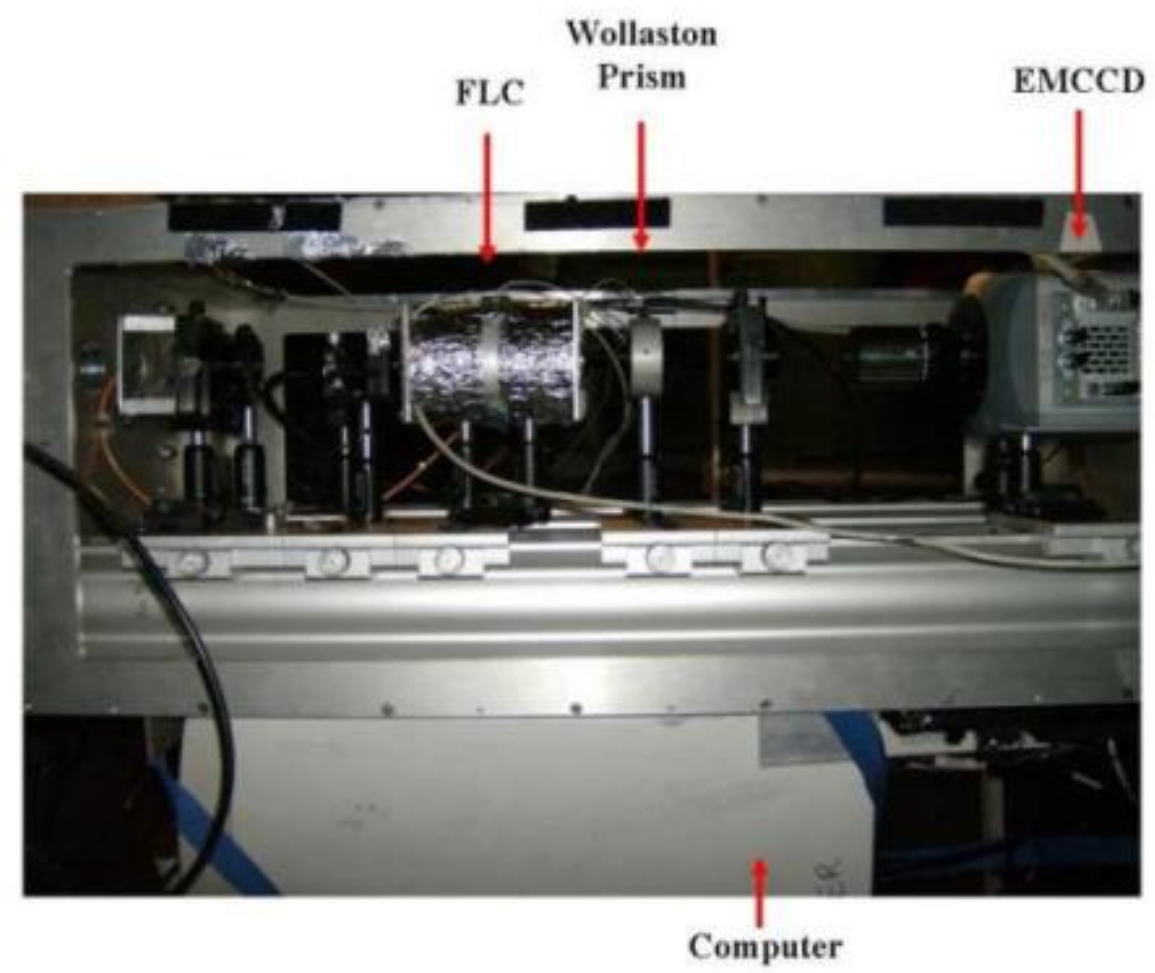

Figure 1.3. PEPPER in Polarimeter mode mounted at Bok Telescope. FLC, Ferro-electric Liquid Crystals; EMCCD, Electron Multiplying Charge-Coupled Device. 


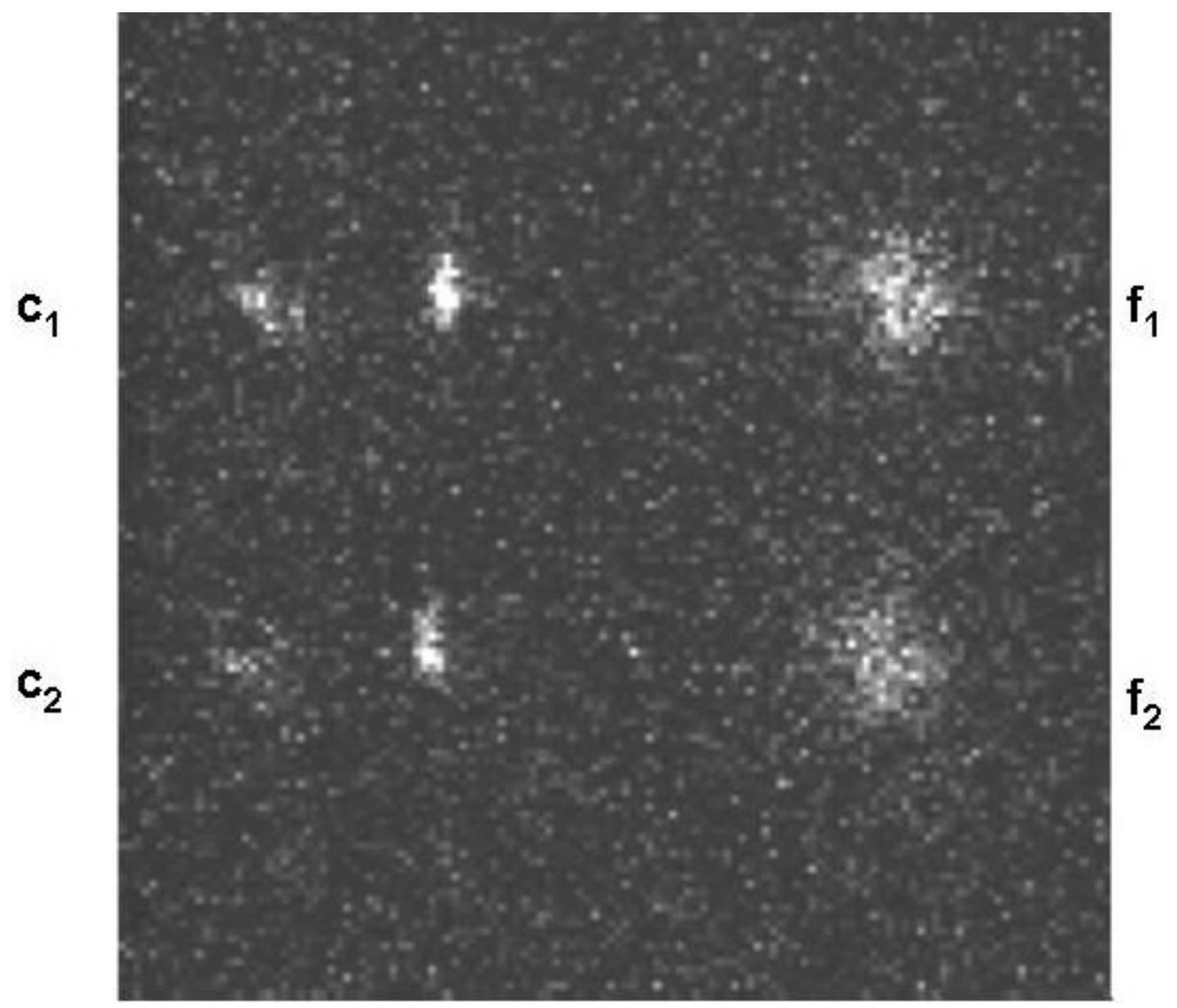

FiguRE 1.4. Raw image of HD209458b (7). c1 and c2, standard star; f1 and f2, parent star. 


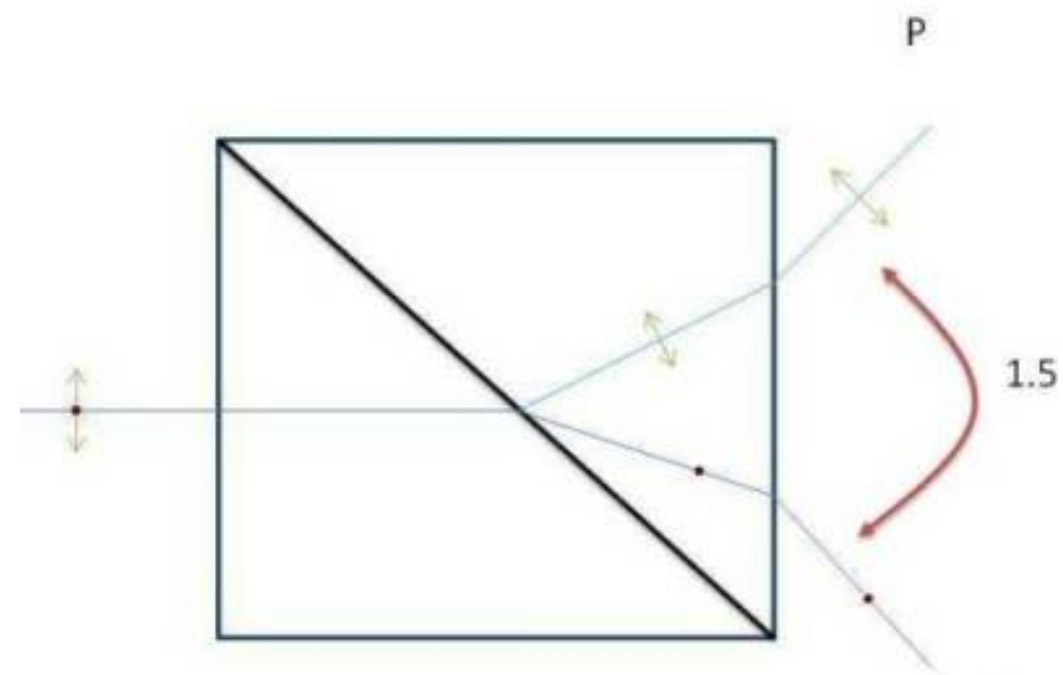

FIGURE 1.5. Wollaston prism diagram showing the incoming beam being split into the two linearly polarized beams, s (into the page) and p (along the page).

are present, the light coming from the $\operatorname{star}(\mathrm{s})$ will be polarized. Therefore, the Wollaston Prism will separate the star's polarization into its $\mathrm{s}$ and $\mathrm{p}$ components. If the light from the star is not being reflected, the Wollaston Prism will have no effect on the incoming light, and so a circumstellar disk will not be detected for that particular $\operatorname{star}(\mathrm{s})$.

\subsection{Photometer}

The Photometer mode of PEPPER was created to monitor a period of a planetary orbit for detection of eclipsing extra-solar planets. This mode works by chopping incoming light at $\mathrm{kHz}$ frequencies to suppress noise. The Polarimeter mode of PEPPER is the base for the Photometer mode. The Photometer mode has a few more necessary components that are not used in the polarimetry mode. A schematic of the Photometer mode is shown in figure 1.7.

The first element in the Photometer mode is the artificial light source. The artificial light source is created by using a white light source which is connected to a fiber optic glued (with UV glue) to a microscope slide and put at the focus of the telescope. 


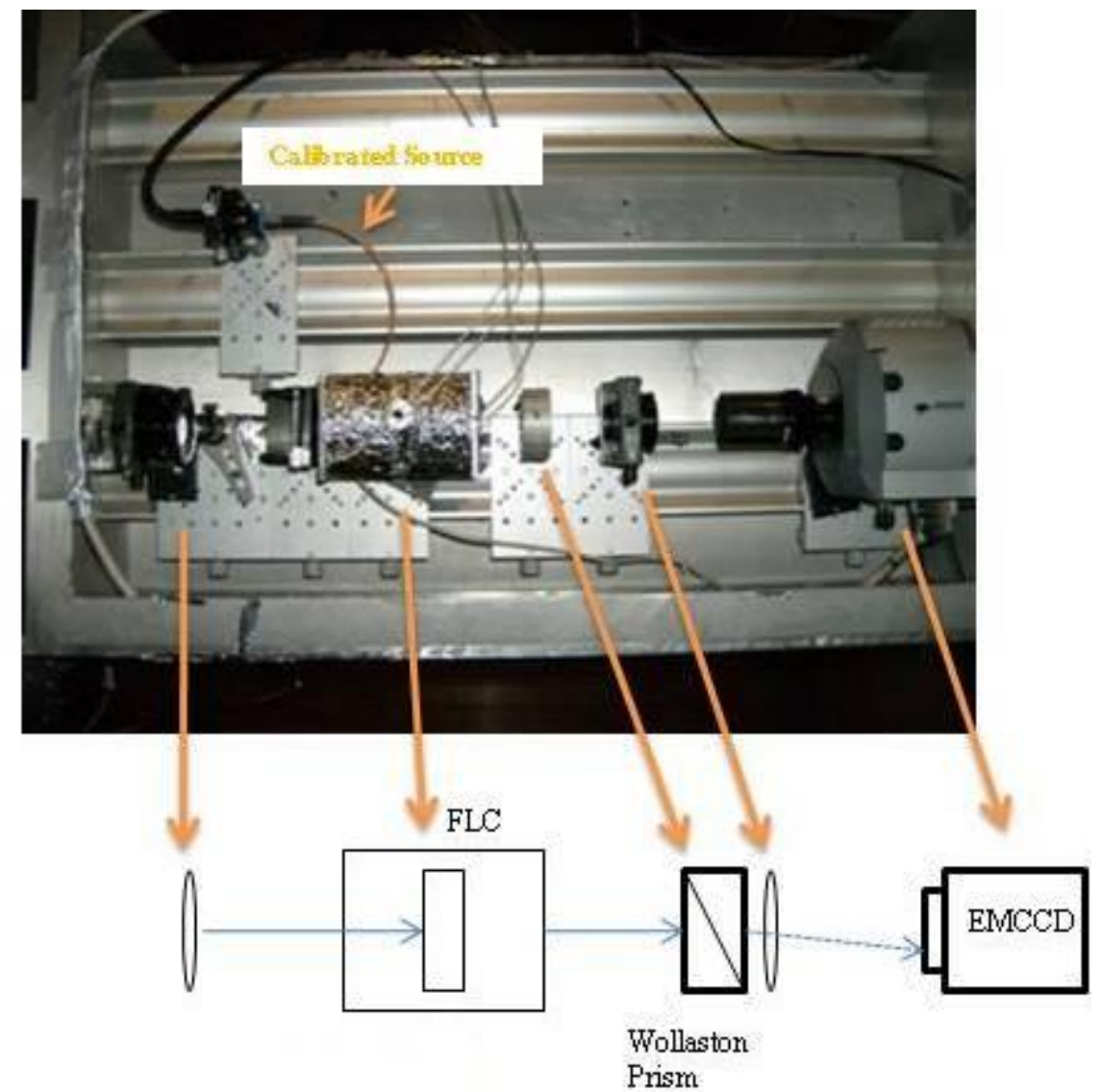

Figure 1.6. Photograph of PEPPER in Polarimeter mode mounted at Bok Telescope, and Polarimeter schematic (below) showing the Ferro-electric Liquid Crystals (FLC), Wollaston Prism, and Electron Multiplying Charge-Coupled Device, (EMCCD) 


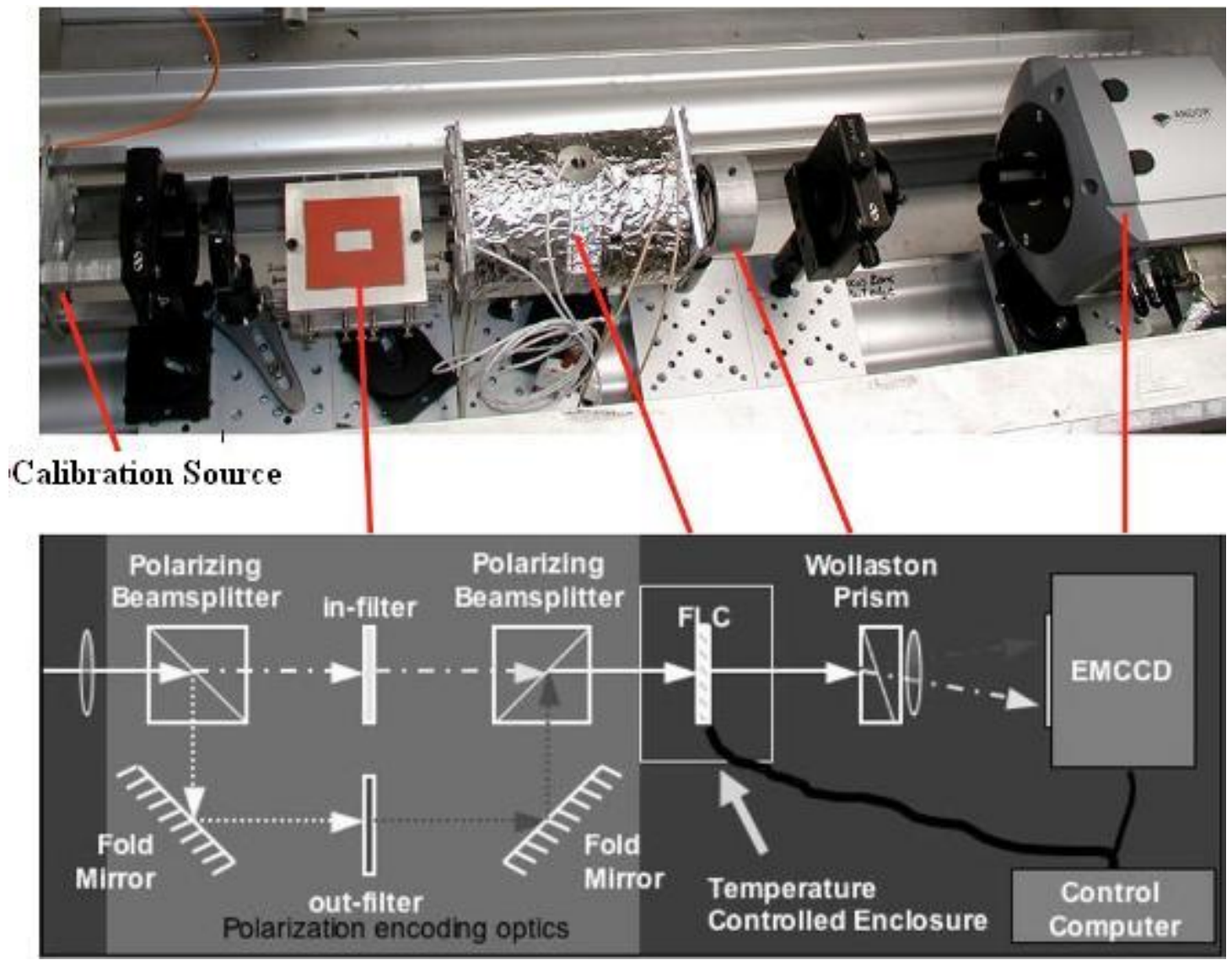

Figure 1.7. While FLC can modulate at $500 \mathrm{~Hz}$, we operated it around $50 \mathrm{~Hz}$. The company name was 'DisplayTech Inc.' (7). 
The artificial light source was used to help in the alignment of the instrument and to mimic a standard star. When using PEPPER, the observer will see the image of the artificial light source and the star (figure 1.4). The light from the artificial source and from the star then go through the aperture into a FLC achromatic rotator. The FLC is used as a polarization retardance modulator at frequencies $>500 \mathrm{~Hz}$, and it modulates faster than the changing atmosphere between two polarization-encoded spectral filters. The FLC was produced by Display Inc. and it has a response time (the minimum time required to change a pixel's brightness) of approximately 100 to $300 \mu \mathrm{s}$ (3). Next, the light goes into the Photometer element of PEPPER. In it, the light passes through a polarizing beam splitter oriented at $45^{\circ}$, splitting the light into orthogonal components. One polarization state passes through an absorption line band pass filter, the other, hits mirror1, passes through a continuum band pass filter before hitting the mirror2 and going through the polarization beam splitter and recombining with the other beam path (2) (see figure 1.8).

Then, the light continues into the Wollaston Prism, where it will be split into $\mathrm{s}$ and p polarization; the light is focused by a focusing lens and detected by the camera. The raw images are acquired, once again, by having the EMCCD set at $120 \mathrm{~Hz}$ per second during the observation of the eclipse. Multiple image cubes are acquired during the time period of the eclipse, and a composite of the images is used to obtain the information of the extra-solar planet. By having a fast acquisition and a high FLC response-time, the noise coming from atmospheric variation and detector can be suppressed. The capability of reducing these errors is very important in order to detect the faint light coming from the extra-solar planet. The raw images contain both the information of the planet and its parent star, thus in order to obtain the information of the extra-solar planet the raw data must be reduced and analyzed, a process described in chapter 2. 


\section{Polarization Encoded Optics}

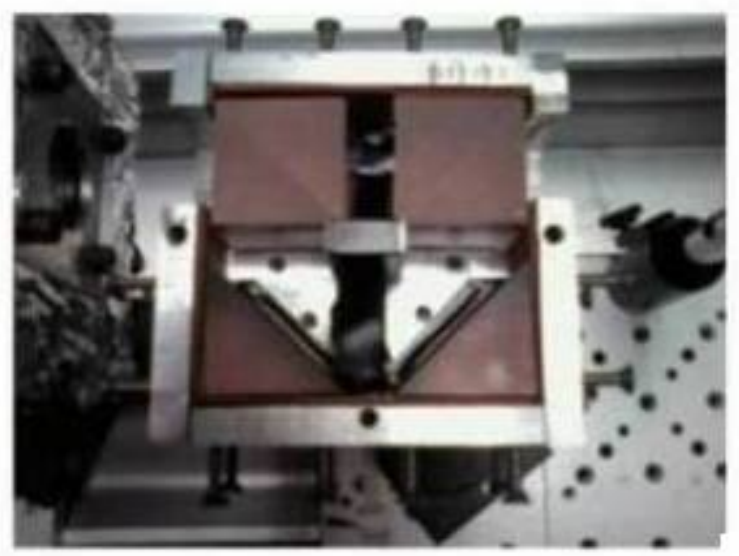

Polarization

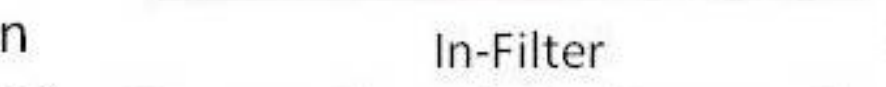

Polarization Beamsplitter

Fold Mirror

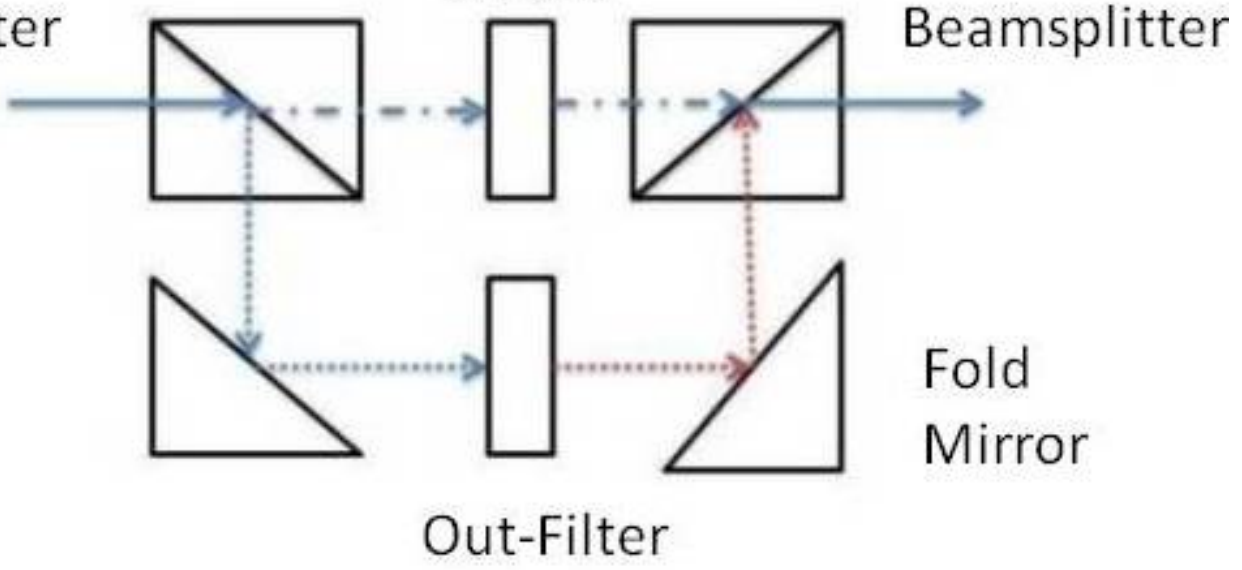

Figure 1.8. Polarization Encoded Optics photograph and schematic. 


\section{Chapter 2}

\section{INSTRUMENT APPLICATIONS}

PEPPER was used primarily at the 90" (2.3m) Bock Telescope located on Steward Observatory Kitt Peak Station, Arizona. During my participation in the project three observing runs were done at the Bock Telescope. One on September $9^{\text {th }}, 2006$, where Dr. Dan Potter and I observed HD209458 and HD210074; and two one-week runs: one on January $28^{\text {th }} 2007$ to February $3^{\text {rd }} 2007$ where I observed HD 32297 , HD281159, HD107156, and HD102870. The second one-week run was done on April $1^{\text {st }}$ to April $7^{\text {th }}$, 2007, and I observed HD 61421, HD107156, HD141569, HD43384, GL338A, Gl250A, and Gl411. Another set of data presented is the data collected by Dr. Dan Potter at the Gemini North Telescope with the Hokupa'a adaptive optics system. A dual channel polarimeter was integrated into the Hokupa'a adaptive optics system mounted on the Gemini North telescope to improve the sensitivity to detect the light scattered by circumstellar material (6). This polarimeter system is very similar to PEPPER, and will help describe the data reduction process. The dual polarimeter technique suppressed the noise introduced by non-repeatable variations of the point spread function, PSF, that limits the sensitivity of non-simultaneous adaptive optics imaging (6). Before describing what was done during the observation runs, here is a brief description of the telescope. The Bok telescope was named after Prof. Bart Bok, who was Stewards Observatory Director from 1966 to 1969. The features utilized during the observation runs were that Bok has an equatorial mount that allows the telescope to track the stars just by rotating one axis that is parallel to the Earth's axis of rotation. This allows the telescope to stay fixed on any object in the sky that has a diurnal motion by moving one axis at a constant speed. To help with the tracking, Bok has two CCD guider cameras, the 90Prime Guider, and the BokCass Guider. The Steward CCD guider cameras are 2 inch high by 4 inch diameter thermoelectrically cooled dewars containing a 1024 x 512 thinned, back illuminated CCDs, which operate at frame-transfer mode (4). These cameras can also be used to take snap shots of what the telescope is looking at, at any given time. Also, the bottom of the Bok telescope, where PEPPER was attached 
to, rotates. PEPPER had a polarization axis fixed to the instrument. To rotate this axis relative to the sky we just rotated the instrument on the back of the telescope, which is equivalent to rotate a HWP. Though there were not utilized during our runs, Bok Telescope has 3 instruments, two optical instruments, the 90prime and Boller \& Chivens Spectrograph, and one infrared instrument, the Steward 256x256 NIR Camera. The specifications of the Bok telescope:

$$
\begin{gathered}
\text { Diameter: } 90.00 " \pm 0.0625 "(2286 \mathrm{~mm}) \\
\text { Conic Constant: }-1.0646 \pm 0.001 \\
\text { Radius of Curvature: } 12281 \mathrm{~mm} \pm 4 \mathrm{~mm} \\
\text { Uncorrected Focal Length: } 6140.5 \mathrm{~mm} \\
\text { Uncorrected Focal Ratio: } \mathrm{f} / 2.69 \\
\text { Corrected Focal Length: } 6829.2 \mathrm{~mm} \\
\text { Corrected Focal Ratio: f/2.98 (4) }
\end{gathered}
$$

Since the focus position of the Bok telescope is dependent on the outside and inside temperatures of the telescope, before an observation run starts the observer needs to check if the system requires small adjustments in focus. Another operational step that needs to be taken before observing starts is the recording of a dark, and a field flat, or dark flat. The dark frames are taken with no external light on the camera. These are taken to calibrate any potential noise static to the detector such as a pattern on the detector made when the detector is read out. It is also referred to as the detector bias levels and they can vary from pixel to pixel thus a calibration frame is necessary. The dark frames should not be sensitive to dust on the detector. The Flat field is taken on an attempt to have even illumination across the focal plane. These flat field frames are used to calibrate the possibly varying pixel-to-pixel quantum efficiency of the detector or varying field dependent throughput of the optical system (i.e. dust near a focal plane). In addition, for the polarimetry runs, a standard star (a star of known magnitude) needs to be recorded. A standard star is used during image processing to normalize the sky around the unknown star. The standard star used was FS-17, faint standard star of magnitude 12 (where magnitude scale goes from the Sun at a magnitude of -26.73 , very bright, to the faintest objects observed in visible light with Hubble Space Telescope of magnitude 30), from the Gemini data February $25^{\text {th }}$, number 399 to 439. During all of the observation runs, PEPPER was installed at the bottom of the telescope, where it moved with the telescope as the 
night progressed. On the September $9^{\text {th }}, 2006$ run, the photometer part of PEPPER was being used to observe a three-hour transit of planet in front of its companion star. For this run PEPPER's camera was set to capture 60,000, 1024x1024 images, at $120 \mathrm{~Hz}$, with an exposure time of 0.00632 seconds, and a gain of 250 . Each exposure contained an image of the star and an image of the standard source. Throughout the two one-week runs, the Polarimetry part of PERRER was used. I observed a variety of stars hoping to detect circumstellar disks in those stars. In those runs the camera was set to $120 \mathrm{~Hz}$ with a duty cycle of 0.5 , and a delay of $0.002 \mathrm{~s}$. The acquisition was set to external, with an exposure time of 0.00632s. The program was set to create unsigned 16 bit integer images. When collecting the data the half wave plate (HWP) was set at angle of $0^{\circ}, 45^{\circ}, 22.5^{\circ}$, and $67.5^{\circ}$. This was done by rotating the bottom of the telescope where PEPPER was attached to at angles of $90^{\circ}, 135^{\circ}, 180^{\circ}$, $225^{\circ}$ degrees. Each acquisition has two polarizations of the star, and each image is composed of 60,000 acquisitions. The examples of polarimetric data analysis are from observations taken at the Gemini North telescope between 2002 and 2003 using the polarimeter inside the Hokupa'a adaptive optics system (6).

\subsection{Polarimetry: Data Reduction and Analysis}

Once the observation runs were complete, the instrument and the data acquired were taken down and back to Steward Observatory for data reduction and analysis. A series of IDL programs were created to reduce and analyze the data. The process of data reduction is different for polarimetry data and photometry data. I was responsible for the analysis of polarization data, and creation of the programs for data reduction was one of my tasks (Program 1 through Program 6). The program starts by taking out known background noise. First, the program subtracts the dark image from the standard star, and then divides the image by the flat. Then, the program creates a bad pixel mask and one image containing all the bad pixels, and applies it to the image, thereby removing the bad pixels from the image. The next step is to account for the background noise in the image that will then be subtracted from the observed star image. This number was found by setting the background as a free parameter

and looking at the behavior of the aperture photometry and the first derivative of the measured photometry with aperture size at large apertures (much greater that 
the Full-Width Half-Mass, FWHM, of the Point Spread Function, PSF). The value of the background where the measured photometry did not change with increasing aperture was used as the best estimate of the background. This number will help to calculate the true magnitude of our unknown star. Once the standard background noise is found, the image processing can begin. The raw data reduction starts by going through a similar process, the bad pixels mask is applied, then the dark frame is subtracted, and finally the flat field is divided. The next step is to find the counts for the unknown star, this is done by finding the center of the star and creating a box that encompasses the star. The counts inside the box are taken. The next step is to subtract the background noise from the image by subtracting the noise found with the standard star. To make the field around the star uniform, a mask is created to encompass the star and the halo around it, and anything outside the mask is set to one. Up to this point, there have been two images of the same object with different polarizations side-by-side. Also, in order to detect the circumstellar disk, the center of the star is over saturated. The next program, program 2, will separate these two images creating 2 sub-frames, sub-frame A and sub-frame B, and correct for the saturation. The separation is done by dividing the images in half and saving them as two separate files. To normalize the center of the observed star is a little more complicated. First the center of the star must be found, and then the program takes a small area around the center of the star and finds the average value of those pixels; the value of the average is then applied to the center of the star. Then, the image is convolved using the convolution command in IDL, and the true center is found by finding the maximum pixel value of the star, which is assumed to be at the center of the star. Program2 then applies this method to both A and B sub-frames. From here, program2 normalizes the background between both sub-frames by creating a small box on the corner of one of the images, and applying it to the other. The images are recombined to create a 540x1024 image, and this is done to all acquisitions to create one clear image of the unknown star, see figure 2.1 for an example. This example is of ROX1-35 which is a binary star system. This data was taken by Dr. Dan Potter with the Hokupa'a adaptive optics system on the Gemini North telescope, the polarimetric observations from Gemini where in the H-band (near IR). Polarization data collected with PEPPER has the same reduction process with a bit of tweak in the Wollaston image separation and scaling. Unfortunately, the PEPPER data was 
not fully reduced due to grant issues. For the ROX1-35 data, the polarimetry images typically had just 20 images per system with 5 taken for each of the 4 HWP rotations. Because ROX1-35 is a binary system the data reduction process has to be done for each star separately since both stars do not necessarily have the same polarization.

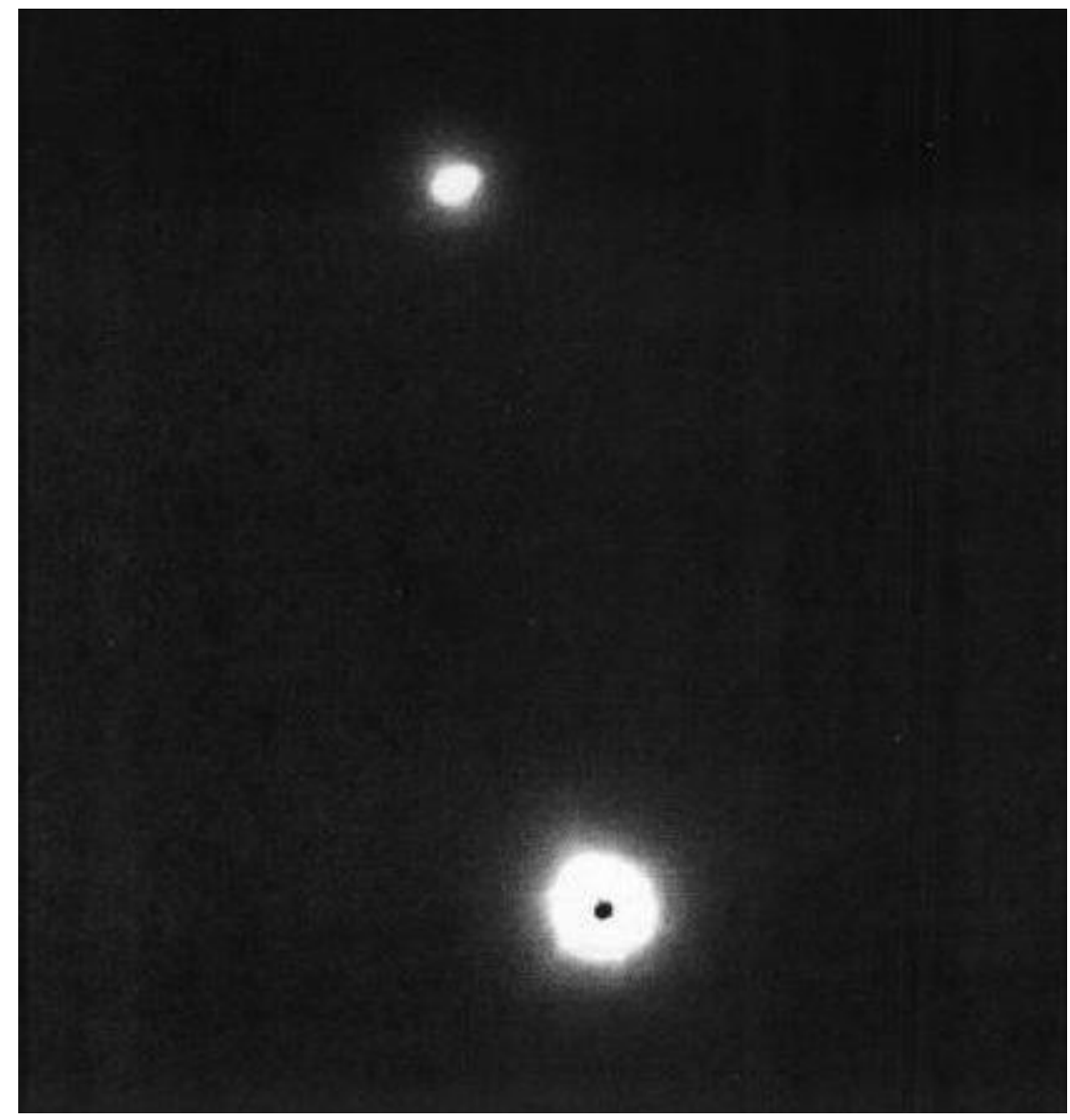

FIgURE 2.1. ROXR1-35 is a binary system. This is an image after the reduction process. After this step the polarization analysis will take place.

During data collection, 5 groups of acquisitions were taken per angle for each object. At this point of the data processing there are 5 images per angle for each object. The next program, program 3, will combine these images to create one. Before adding the images, the program aligns the images by using the shift command, which gives an approximate alignment. To get the exact alignment, the interpolate command is used; this command does a bilinear interpolation of a two dimensional image. From here the images are added, or subtracted using the Stokes Parameters 
to create the polarized images. The images are added in the following way:

$$
\begin{gathered}
H W P=0^{\circ}: Q=I_{0^{\circ}}-I_{90^{\circ}} ; \\
H W P=45^{\circ}: Q=I_{90^{\circ}}-I_{0^{\circ}} ; \\
H W P=22.5^{\circ}: \quad U=I_{45^{\circ}}-I_{135^{\circ}} ; \\
H W P=67.5^{\circ}: \quad U=I_{135^{\circ}}-I_{45^{\circ}} .
\end{gathered}
$$

Where a 22.5 degree rotation on the HWP is the equivalent of rotating the instrument 45 degrees. This process is done for all four different angles for each object, what is left are two $\mathrm{Q}$ images and two $\mathrm{U}$ images. To combine these two images, the direction of polarization needs to be determined, which is achieved by looking at the current processed image, see figure 2.2 below. The polarization intensity, PI, polarization amplitude, $\mathrm{P}$, and polarization angle, Theta, can be calculated using the following equations:

$$
\begin{gathered}
\mathrm{PI}=\sqrt{Q^{2}+U^{2}} \\
\mathrm{P}=\sqrt{(Q / I Q)^{2}+(U / I U)^{2}} \\
\text { Theta }=1 / 2 * \operatorname{atan}(Q / U)
\end{gathered}
$$

Where IQ is equal to $I_{0^{\circ}}+I_{90^{\circ}}$, and IU is equal to $U=I_{45^{\circ}}+I_{135^{\circ}}$

The two Q images are added if the polarization is in the same direction, and subtracted if the polarization is in the opposite direction. The same process is used to combine the $\mathrm{U}$ polarized images. During the addition and subtraction, the shift and interpolate commands are used again to insure that the objects are centered. From here, the total Q polarized images and the total U polarized images are aligned to each other and combined by taking the square root $\left(Q^{2}+U^{2}\right)$ to create the final image. Also, the intensity, magnitude, average, and standard deviation of the star are calculated. This process and analysis creates the final image (see figure 2.3 for final image of ROX1-35), which is a positive detection for circumstellar disks.

Figure 2.3 is a high resolution (FWHM 75mas) HST WFPC2 image of SR24 $\mathrm{N}(\mathrm{ab}) / \mathrm{S}$ in the F606W filter (shown on the left), with a surface brightness scale in mag / arcsec2. The H-band polarized intensity image (FHWM 85mas) is shown on the right, with a similar surface brightness scale. The regions within 0.2 " of the stellar photocenters were saturated in these data. Intensity images showing SR $24 \mathrm{~N}(\mathrm{ab})$ as a 0.1" binary and SR24 S as a single star have been overlaid on these masked-out 


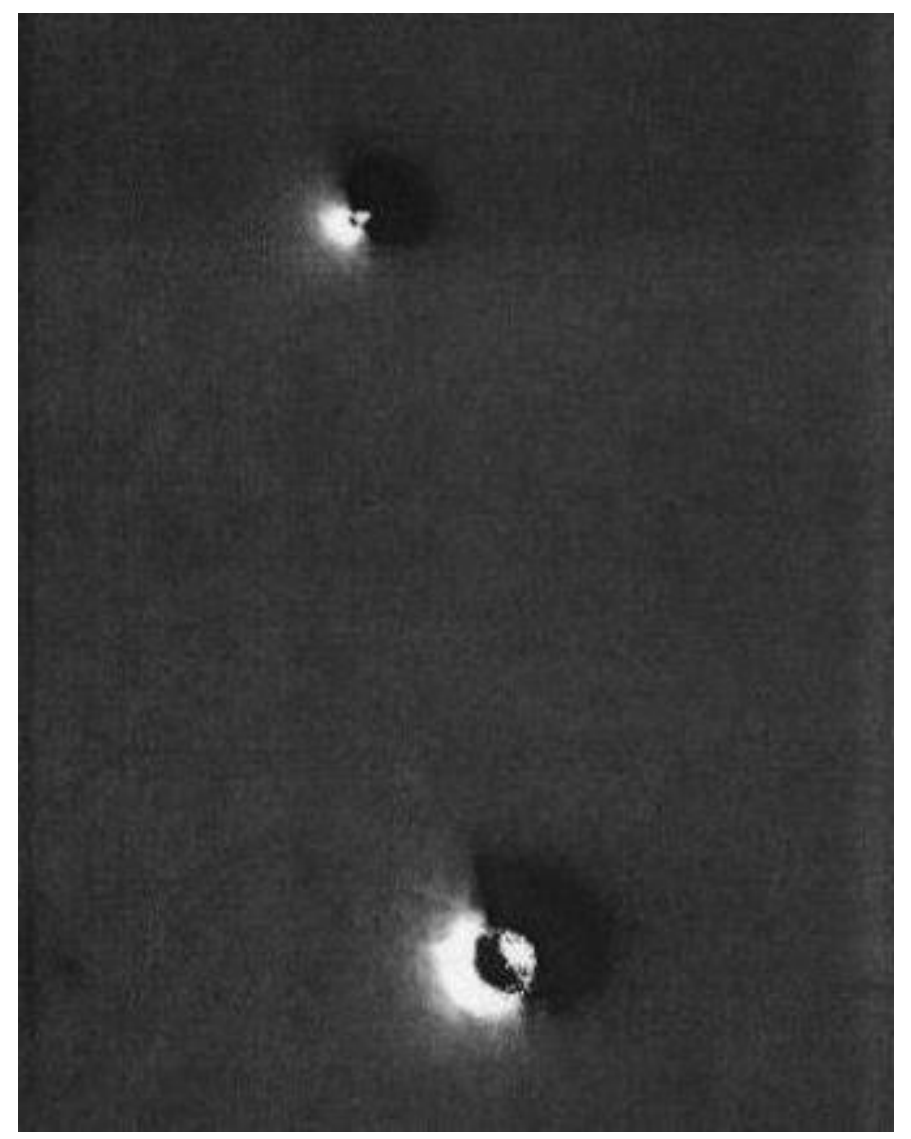

FIgURE 2.2. ROXR1-35 processed before combining all the polarizations. Note: the center of the stars are still saturated. 


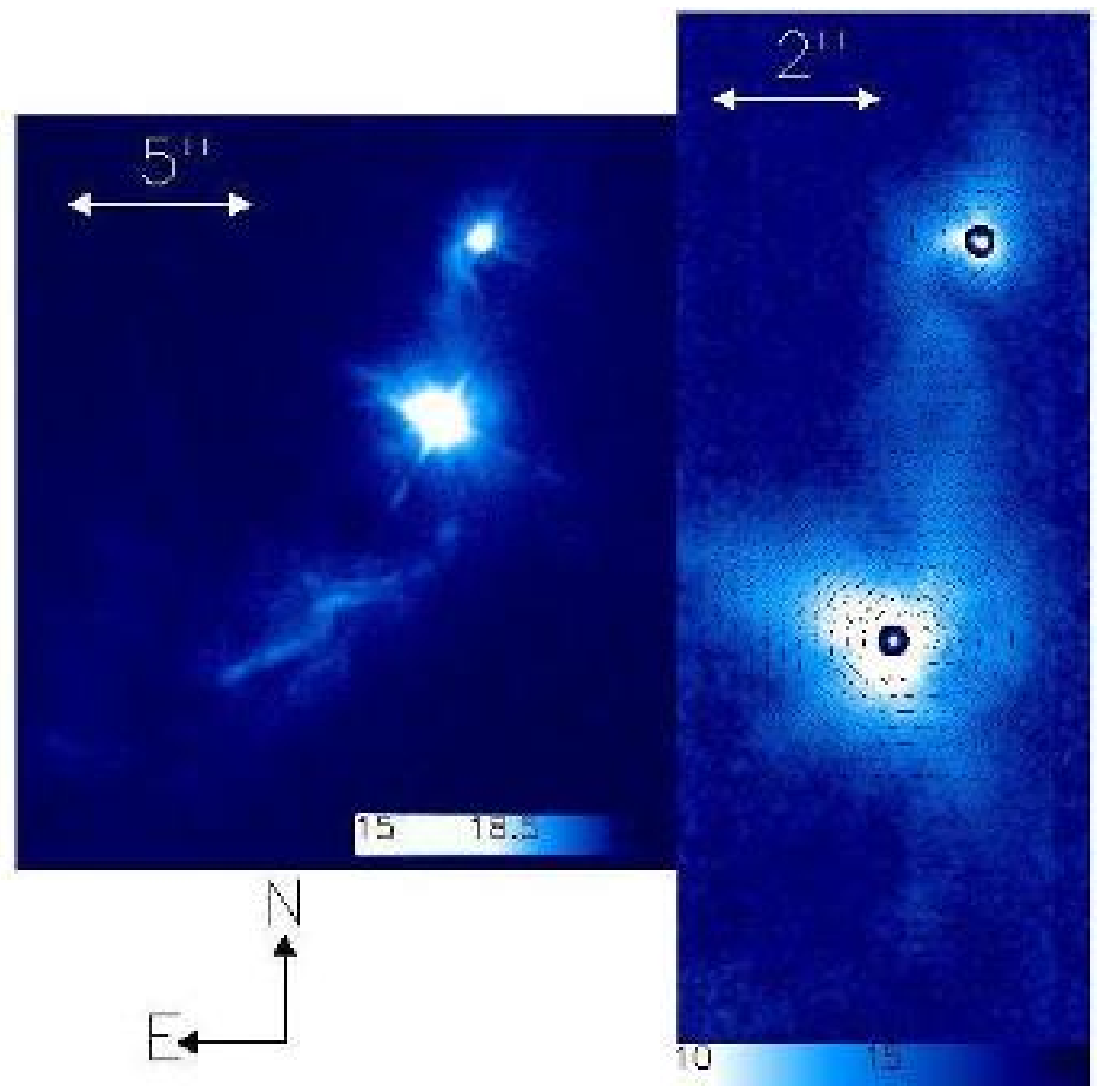

Figure 2.3. ROXR1-35 final processed image (5). Different intensities are demonstrated by the shades of blue color. 
saturated regions. The overlaid polarization vectors are the result of binning over 0.2 " $\mathrm{x} 0.2$ " regions. This process was used to reduce and analyze all the polarimetry data. A symmetrical polarization pattern needs to be observed in order to confirm circumstellar disk detection. Figure 2.4 is an example of what to expect when looking for circumstellar disks; on the left is the result of the analysis of the nearest CTTS, TW Hydrae, which shows a near-perfect example of a center of symmetric scattering pattern from a circumstellar disk, with a $45^{\circ}$ position angle shift between $\mathrm{Q}$ and $\mathrm{U}$ images (5) which would be considered a detection of circumstellar disks; on the right, is an example of what is expected to be recorded when there is no detection of a circumstellar disk.

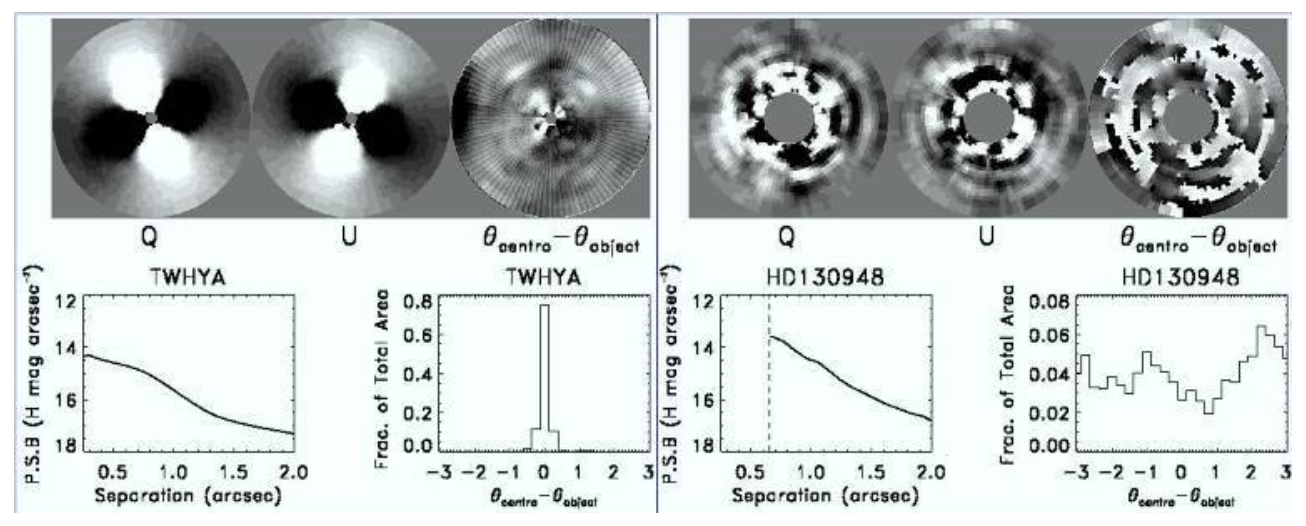

FIGURE 2.4. Left, detection of circumstellar disk, right non-detection of circumstellar disk (5).

The three images, on the left side of figure 2.4, show the detection of a circumstellar disk. The Stokes Q and U images where produced by the Point Spread Functionvariation-insensitive dual imaging technique, which acts as an effective means for detecting circumstellar dust illuminated by a central source (6). The image on the right of this group is a gray scale display of the deviation between the measured polarization angles produced by a perfect centrosymmetric scattering; the left and middle images are the total $\mathrm{Q}$ and $\mathrm{U}$ polarizations of the perfect centrosysmmetric scattering. The plot on the lower left hand corner shows the radial profile of the polarized surface brightness. The right hand plot is a histogram of the number of bins at a given deviation angle from the center of symmetry pattern. The vertical axis shows the fraction of total bins in the deviation range noted by the horizontal 
axis (5). On the right hand side of figure 2.4 there are equivalent images and plots for the non-detection case. As shown there is no symmetry in the images or resulting plot (bottom right hand side). Figure 2.5 is another example of data collected that had no circumstellar disk detection.

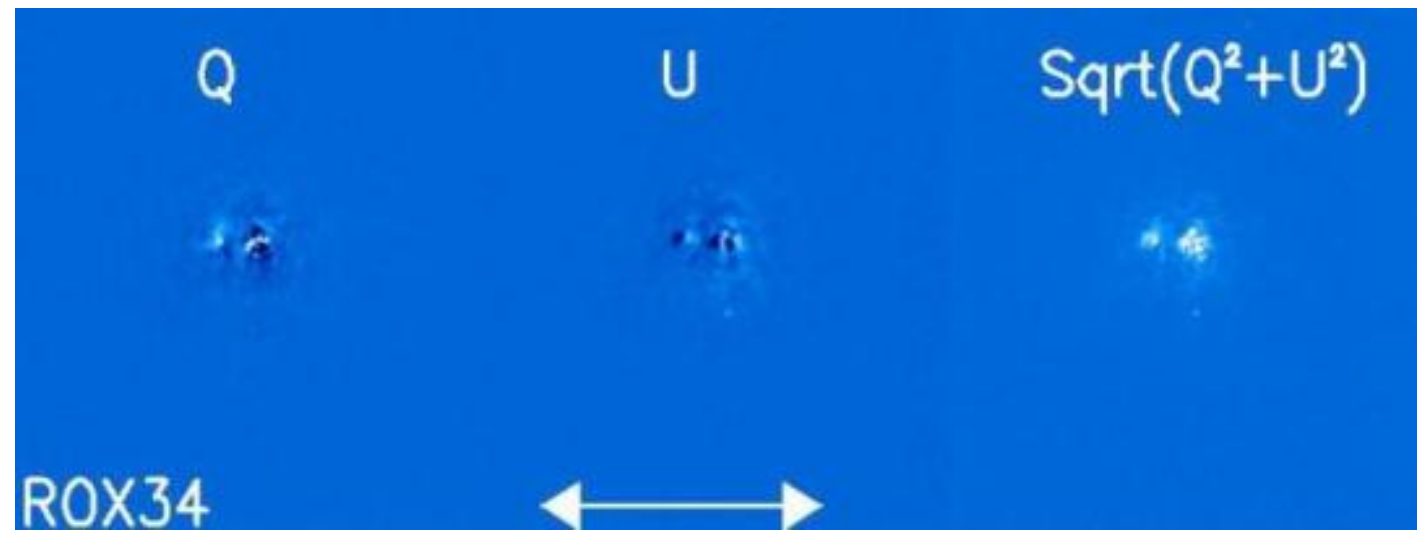

Figure 2.5. This is the binary system ROX-34. It has no polarization symmetry, and so no circumstrellar disk detection. The color of the image is artificial (5).

\subsection{Photometry: Data Reduction and Analysis}

During the observations performed on September $9^{t h}, 2006$ one of the objects observed was the transit of HD209458b (Osiris an Extra-solar Planet Candidate) over its parent star. We expected the parent star to be a $7^{\text {th }}$ magnitude star to have about $2 \times 10^{6}$ photons/second available for observation using a 2-meter telescope with a $\Delta \lambda=5 \mathrm{~nm}$ narrow band filter. The predicted photon noise limit is about $1 x 10^{-5}$ accuracy for the course of approximately 2 hours (7). Figure 2.6 has a schematic of the planet's orbit around the star, with the different wavelength ranges, which will be explained next.

Figure 2.6 illustrates the relative illumination of the planet as seen from the f1 filter (for wavelengths $587 \mathrm{~nm}$ to $592 \mathrm{~nm}$, red) and f2 filter (for wavelengths $598 \mathrm{~nm}$ to about $603 \mathrm{~nm}$, blue) (figure 2.6, and figure 2.7). f2 is the out-of-band filter which should reflect light from the star more efficiently than from f1, which is shown as gray color on the illumination side due to the absorption by sodium (or potassium). 

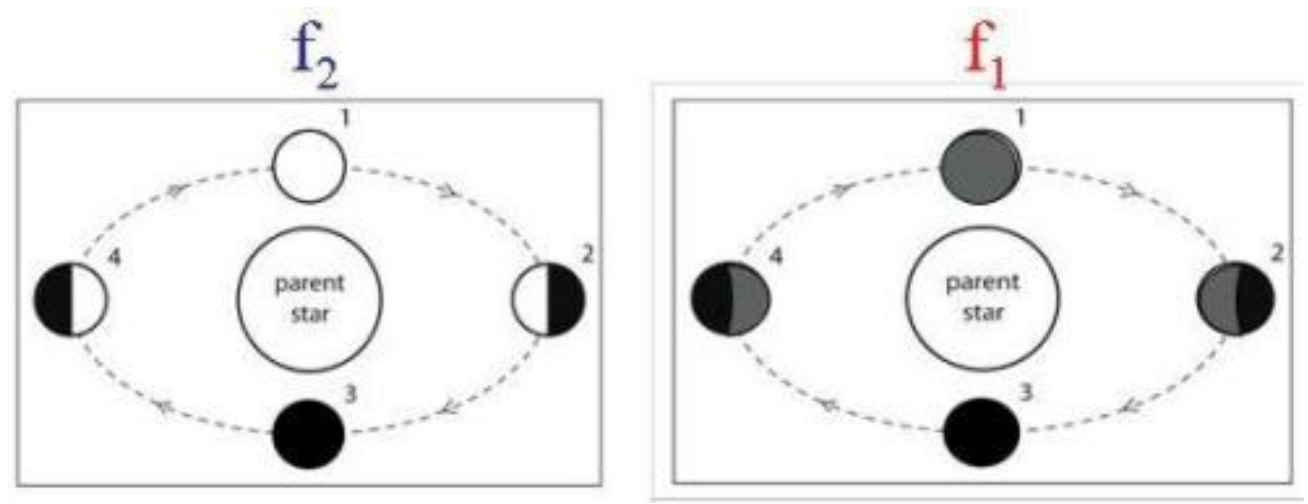

Figure 2.6. Model of HD209458b transit around its primary star (7). f1 filterwavelengths $587 \mathrm{~nm}$ to $592 \mathrm{~nm}$; f2 filter-wavelengths of $598 \mathrm{~nm}$ to about $603 \mathrm{~nm}$.

There should be relatively no difference when the planet is in position 3 (figure 2.6), however, significant differences should be expected when the planet is at position 1 .

In order to get the complete orbit of the star another observation run was done by Dr. Dan Potter on October $9^{t h}$ through $12^{t h}, 2006$, at the Bok Telescope. Figure 2.8 is an example of the type of raw data that was collected during this run.

The image on figure 2.8 clearly shows the standard star, created by the artificial light source ( $\mathrm{c} 1$ and $\mathrm{c} 2$, on the left the image) and the star being observed the parent star (f1 and $\mathrm{f} 2$, on the right of the image). The filters were centered at a wavelength of $770 \mathrm{~nm}$ and $800 \mathrm{~nm}$, with incremental wavelength changes of $10 \mathrm{~nm}$ (7). In order to calibrate the images two equations were used:

SignalNumerator $=\left(\left(f_{1+}-f_{1-}\right)-\left(f_{2+}-f_{2-}\right)\right) /\left(f_{1+}+f_{1-}+f_{2+}+f 2-4\right.$ darkf $)$

SignalDenominator $=\left(\left(c_{1+}-c_{1-}\right)-\left(c_{2+}-c_{2-}\right)\right) /\left(c_{1+}+c_{1-}+c_{2+}+c_{2-}+4\right.$ darkc $)$

Where the 1 and 2 are for the two positions on the detector from the Wollaston Prism split. The $+/$ - is representing the state of the FLC (7). Each state acts as a modulator on the filter such that:

$$
c_{1+}=c_{2-} \text { or } c_{1-}=c_{2+}
$$

Where: $c_{1+}$ is opposite filter of $c_{2+}$, and $c_{1-}$ is opposite filter of $c_{2-}$

These equations resolve the variability from the noise sources in the instrument and from the atmosphere (7). With the information gained from the calibrations, the 


\section{Models for HD209458b}

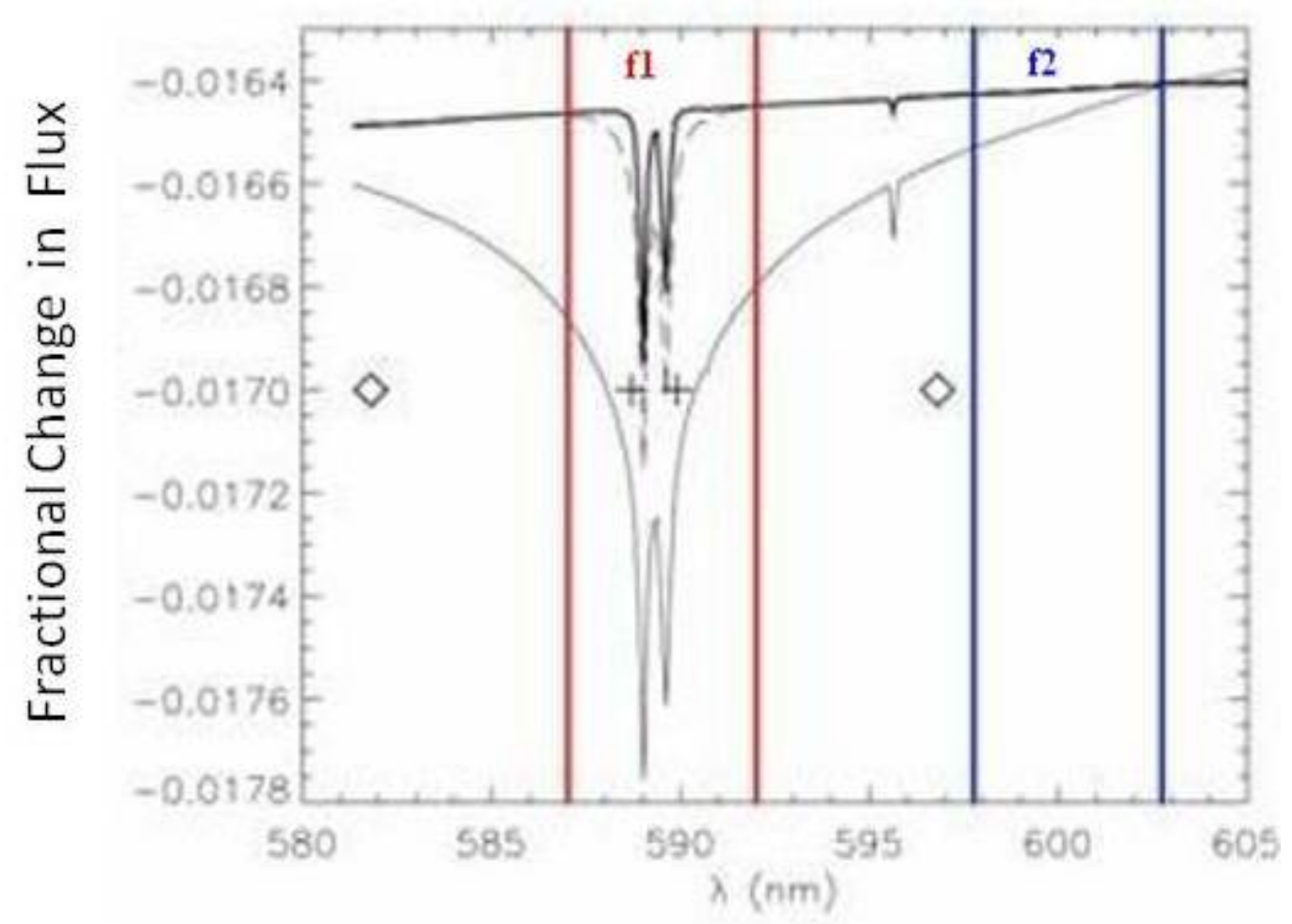

Figure 2.7. Model for HD209458b(7). f1 filter: red, $587 \mathrm{~nm}$ - $592 \mathrm{~nm}$; f2 filter: blue, $598 \mathrm{~nm}-603 \mathrm{~nm}$. The absorption feature shown in the graph is the sodium D line at $589 \mathrm{~nm}$. However, our observations used a similar line caused by potassium in the planet's atmosphere at $700 \mathrm{~nm}$. The absorption band is in the planetary atmosphere. In this band the planet would look black regardless of the phase angle compared to the out-of-band line. It also shows the sodium Fraunhofer lines in star. 


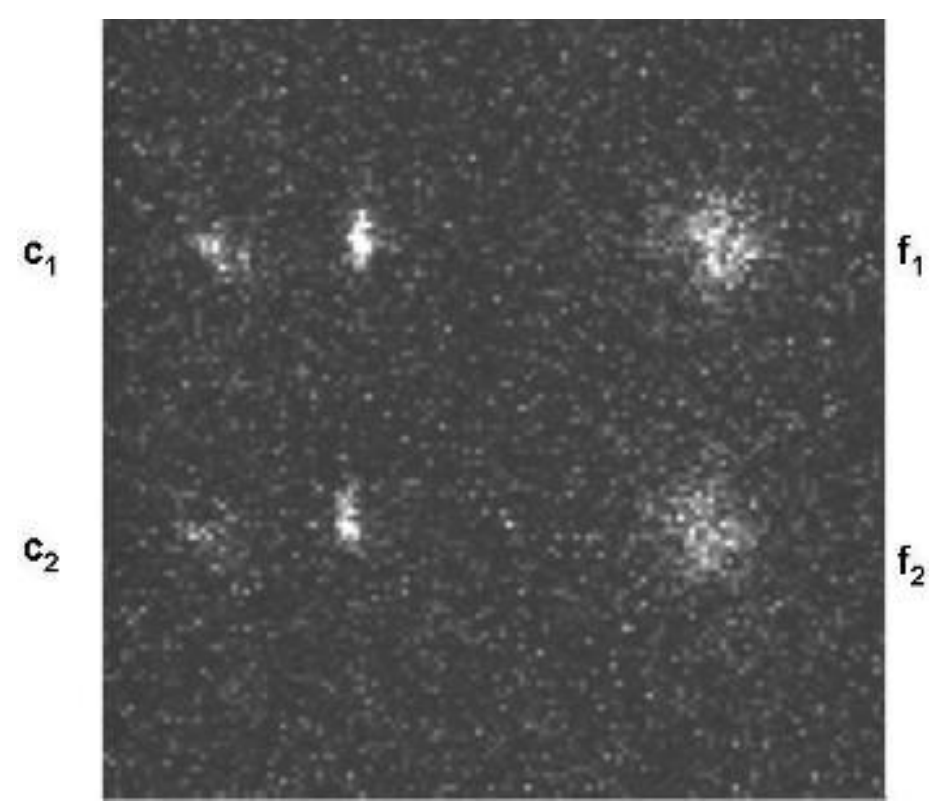

Figure 2.8. Raw image of HD209458b (7). c1 and c2, standard star; f1 and f2, parent star.

differential signal can be obtained by dividing the numerator and the denominator. The next graph (figure 2.9) was a plot created from the data collected during the October run. The plot is comprised of over 5 million images that were analyzed. The curve of the plot is due to the two narrow band filters having a differential extinction with airmass of approximately $6 \times 10^{-4}$ over 2 airmasses; the planet signal was calculated to be about $7 \times 10^{-5}$ (figure 2.9) (7). The plot shows the normalized differential signal of Osiris from the beginning of the eclipse to the end. As the plot shows there was an increase in signal right after the eclipse began. This means that by using PEPPER with a reference filter set at a wavelength of $770 \mathrm{~nm}$ we can detect light reflected from Osiris at $800 \mathrm{~nm}$ at approximately $3 \sigma(7)$. 


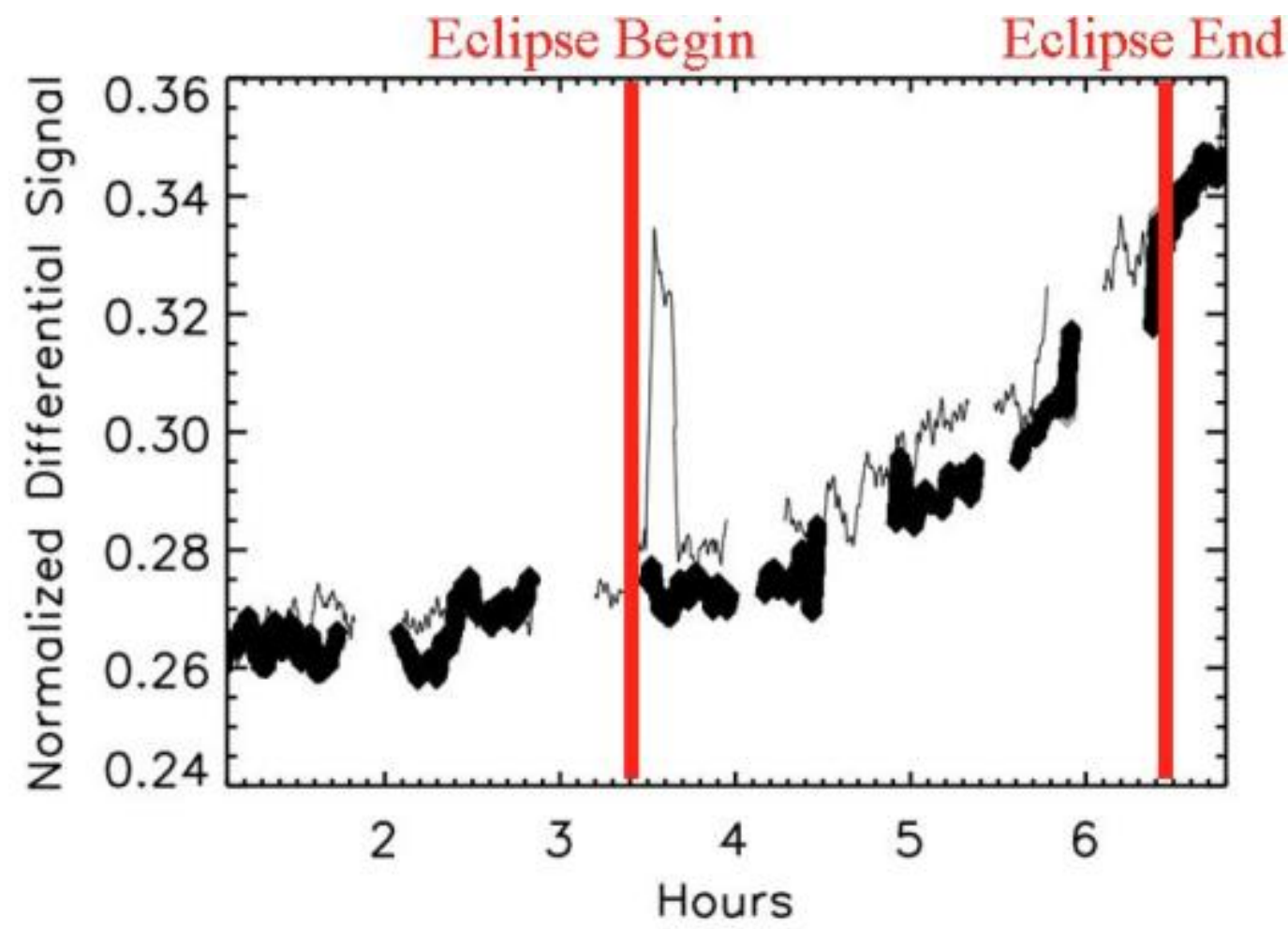

Figure 2.9. Differential Signal for Osiris (HD209458b), an extra-solar planet candidate (7). The bold line is HD209458b on a night where there was a secondary eclipse while the thin line indicates a night when there was none. Signal gaps correspond to periods when standard star data was being acquired during the run. 


\section{CONCLUSION}

The Observations made at the Bok Telescope at Kitt Peak Arizona, validated PEPPER, Polarization-Encoding differential Photometer and PolarimetER. As shown earlier by Dr. Dan Potter and in this report, this instrument can achieve near photon noise limit results in the laboratory and on sky observations to levels of 1 part in $10^{5}$. In addition, PEPPER is capable of acquiring a great amount of images at a high rate $(120 \mathrm{~Hz}$ per second and the EMCCD can go at a higher rate if needed), and it has a high FLC response time. This capability allows for atmospheric variation and detector noise suppression, which gives PEPPER the capability of recording high resolution data without the use of adaptive optics, and a space telescope. PEPPER can detect the transit of a planet around its parent star, such as for the planet HD209458b, which was detected at about $3 \sigma$ at $800 \mathrm{~nm}$ using this approach with a reference filter set of $700 \mathrm{~nm}$. Also, it can collect the data needed to determine whether or not a star possesses a circumstellar disk. The examples shown were of ROXR1-35, a binary star system in which both stars have a circumstellar disk, and ROX-34, also a binary star system, which had no circumstellar disk detection. 


\section{REFERENCES}

[1] Matthew C. Graham, Dan E. Potter , and Laird Close: "Polarimetry mode of PEPPER: A High Speed Imaging Polarimeter" Ground-based Instrumentation for Astronomy SPIE Meeting poster 2004.

[2] Matthew C. Graham, Dan E. Potter, and Laird Close: "PEPPER: a photometer designed for the direct detection of extrasolar planets" Ground-based Instrumentation for Astronomy Alan F. Moorwood, Masanori Iye, ed.,Proc. SPIE 5492, p. 545, 2004.

[3] Dan E. Potter: "A Zero Read Noise, High Speed, High Sensitivity Photometer and Polarimeter: An Instrument for the direct detection of Inner Extrasolar Planets and Debris Disks", First Year Progress Report.

[4] Jim Burge: "Optical Design and specifications for the 90-in Prime Focus Corrector Part 1: specifications and tolerances", Revision E-2, October 26, 2000.

[5] Catarina Ubach, Dan E. Potter: "Dual Imaging Polarimetry of Young Stars in Rho Ophiuchus", AAS Seattle Meeting, poster $200 \%$.

[6] Dan Potter: "A Search for Debris Disks around Young Solar Analog Stars using an Adaptive Optics Dual Imaging Polarimeter" PhD Thesis, UNIVERSITY OF HAWAI'I, Source DAI-B 64/05, p. 2228, Nov 2003, 160 pages, 2003.

[7] Dan Potter: "PEPPER: Observations of the HD209458 Eclipse", ASS Seattle Meeting, presentation $200 \%$.

[8] Dan E. Potter: "The Performance and Data Reduction Techniques for an Adaptive Optics Dual Imaging Polarimeter", Steward Observatory, 933 North Cherry Ave, Tucson, AZ 85721. 